\title{
Uso consuntivo del agua. El caso de los lavaderos de automóviles en la ciudad de Pilar, Paraguay
}

\author{
Isabel Benítez de Candia ${ }^{1}$ \\ ibenitezdecandia@gmail.com \\ Nicanor Antola Segovia ${ }^{2}$ \\ nsegovia@aplicadas.edu.py \\ Instituto de Ciencias Ambientales. \\ Facultad de Ciencias Aplicadas. Universidad Nacional de Pilar \\ Pilar - Paraguay
}

\section{RESUMEN}

El agua es un recurso fundamental para la supervivencia, por lo que su aprovechamiento debe ser racional, previniendo derroche, contaminación y degradación para evitar alteraciones significativas en la dinámica del ecosistema. La investigación analizó el uso del agua en los lavaderos de automóviles de la ciudad de Pilar a fin de identificar la fuente de origen, determinar el tratamiento y disposición final de efluentes, así como el cumplimiento de normativas ambientales vigentes. El estudio descriptivo, utilizó el enfoque mixto, con diseño observacional, transversal. De la población basada en el registro municipal, se tomó una muestra aleatoria, utilizando técnicas de encuesta y observación, apoyadas en cuestionario y guía de observación. La fuente mayormente utilizada es de agua subterránea y en menor proporción, de la red de servicio sanitario (ESSAP). No se cumple con el sometimiento a Evaluación de Impacto Ambiental ni inscripción en el Registro Nacional de Uso y Aprovechamiento de Recursos Hídricos. El tratamiento de efluentes es deficiente y su disposición se realiza en alcantarillados pluviales, cloacales y en cursos naturales de agua. Se evidencia el uso consuntivo del agua en esta actividad, ya que se devuelve en condiciones distintas a las extraídas y se pierde, sin posibilidad de reutilización.

Palabras clave: agua, uso consuntivo, recursos hídricos, aprovechamiento, lavaderos.

\footnotetext{
${ }^{1}$ Abogada. Especialista en Evaluación de Impacto y Gestión Ambiental por la Universidad Nacional de Pilar. Gestor Cultural y Ambiental por la Universidad Nacional de Itapúa, Misiones. Docente de las cátedras Legislación Ambiental, Auditoría Ambiental y Recuperación del Patrimonio Cultural y Ambiental de la Facultad de Ciencias Aplicadas de la Universidad Nacional de Pilar. Pilar- Paraguay. E-mail: isabeniezg@hotmail.com

${ }^{2}$ Licenciado en Ciencias Ambientales por la Universidad Nacional de Pilar. Especialista en Gestión Integral del agua por la Universidad Europea del Atlántico. Gestor Cultural y Ambiental por la Universidad Nacional de Itapúa, Misiones. Docente de las cátedras Biología General y Microbiología de la Facultad de Ciencias Aplicadas de la Universidad Nacional de Pilar. Pilar - Paraguay. E-mail: nsegovia@aplicadas.edu.py
} 


\title{
Analysis of the environmental legal framework applicable to the protection and conservation of urban wetlands in the city of Pilar
}

\begin{abstract}
Water is a fundamental resource for survival, so its use must be rational, preventing waste, pollution and degradation to avoid significant alterations in the dynamics of the ecosystem. The investigation analyzed the use of water in the car washes in the city of Pilar in order to identify the source of origin, determine the treatment and final disposal of effluents, as well as compliance with current environmental regulations. The descriptive study used a mixed approach, with an observational, cross-sectional design. From the population based on the municipal registry, a random sample was taken, using survey and observation techniques, supported by a questionnaire and observation guide. The main source used is groundwater and, to a lesser extent, from the sanitary service network (ESSAP). The submission to the Environmental Impact Assessment or registration in the National Registry for the Use and Exploitation of Water Resources is not fulfilled. Effluent treatment is deficient and its disposal is carried out in storm sewers, sewers and natural water courses. The consumptive use of water in this activity is evidenced, since it is returned in conditions other than those extracted and is lost, without the possibility of reuse.
\end{abstract}

Keywords: water, consumptive use, water resources, exploitation, laundries

Artículo recibido: 03 nov. 2020 Aceptado para publicación: 07 dic. 2020 Correspondencia ibenitezdecandia@gmail.com Conflictos de Interés: Ninguna que declarar 


\section{INTRODUCCIÓN}

El Paraguay es un país potencialmente rico en recursos hídricos, pero este recurso es irregular en su forma de presentarse en el tiempo y en el espacio, fácilmente vulnerable y susceptible de usos sucesivos. Al respecto, Navascués (2013) refiere que el agua constituye un recurso unitario, que se renueva a través del ciclo hidrológico y que conserva, a efectos prácticos, una magnitud casi constante dentro de cada una de las cuencas hidrográficas.

El sistema de humedales Paraguay-Paraná es una de las mayores reservas de agua dulce y biodiversidad del mundo, abarca territorios de Argentina, Brasil, Bolivia, Paraguay y Uruguay. Los esteros de Ñeembucú constituyen uno de los principales humedales de este sistema (FAO, 2015): Este sistema de humedales, es el mayor corredor de humedales del mundo, va desde el Pantanal de Mato Grosso, hasta el río de la Plata. Cumple funciones como mitigar grandes inundaciones y sequías, recarga de acuíferos, provisión de alimentos, mantener áreas para la cría de peces, facilitar la agricultura y la ganadería y proveer agua dulce; con lo cual genera trabajo y renta.

$\mathrm{El}$ acceso al agua potable es un derecho humano indispensable para una vida digna (ONU, 2002), pero, además del uso para consumo de la población, se debe mejorar su gestión en otros usos, como el agrícola (riego, ganadería, acuicultura) y el industrial. Por ello surge el debate nacional sobre el marco jurídico, institucional y de políticas implementadas por el Estado para el uso y gestión del agua.

En este desafío, el PNUD (2006) apunta a asegurar la sustentabilidad ambiental en general y del agua en particular y, por una cuestión ética, enfatiza en la necesidad de promover con suficiente fuerza la responsabilidad intergeneracional mediante un uso responsable de los recursos naturales y la conservación del ambiente.

La FAO indica que los principales usuarios del agua en el Paraguay son la población, la ganadería, la agricultura con riego y la industria. Asimismo, menciona entre los usos no consuntivos, al de las represas hidroeléctricas y la navegación que depende de los niveles del río. Este organismo internacional analiza el uso extractivo o consuntivo y presenta los siguientes datos: La extracción hídrica total nacional para el 2012 alcanzó los $2.413 \mathrm{~km}^{3}$, destacando el sector agrícola con una extracción estimada de $1.897 \mathrm{~km}^{3}$ (de los cuales $0.297 \mathrm{~km}^{3}$ corresponden a la ganadería), equivalente al 79 por ciento del total de las extracciones, la extracción municipal alcanzó los $0.362 \mathrm{~km}^{3}$, o el 15 por ciento del total, 
y la industria (en el departamento Central, ya que no se disponen datos del resto del país) alcanzó una extracción de $0.154 \mathrm{~km}^{3}$, o el 6 por ciento del total de las extracciones (FAO, 2015).

La investigación aborda el uso industrial del agua, en particular, aquel que involucra a los lavaderos de automóviles de la ciudad de Pilar, Departamento de Neembucú en el año 2019, ciudad que posee diversos cursos de agua naturales, los cuales tienen como función mantener la calidad y el caudal de las aguas en las cuencas hidrográficas.

El proceso del lavado de automóviles requiere un consumo importante de agua y genera un vertido de carácter industrial. Las fuentes de suministros pueden provenir de agua subterránea como superficial y la cantidad de agua utilizada varía según el tipo de sistema para el lavado de vehículos (manual, eléctrico o electromecánico), el tiempo empleado para cada lavado y la cantidad de vehículos involucrados.

Tafur (2017) indica que, para calcular cuánta agua es utilizada de manera directa o indirecta, se sugiere el empleo de la huella hídrica; este es un indicador que permite calcular el volumen de agua dulce que consume un habitante o una comunidad o ya sea una industria en la fabricación de un producto. Implementar el uso de agua lluvia, por lo cual el ahorro de agua permitirá la racionalización del agua (Mendieta, 2016), al igual que contar con colectores para evitar el desperdicio de las primeras aguas de lavado, que pueden ser reutilizados si se decanta de manera eficiente.

En cuanto a las aguas residuales, requieren un tratamiento para eliminar parte de su carga contaminante, a fin de posibilitar su reutilización y minimizar el impacto en el balance hídrico. Busca mejorar la calidad de las aguas, que va desde la decantación de solidos hasta la eliminación de contaminantes como nitratos, fosfatos, etc., incluyendo la desinfección. De no realizarse el tratamiento, provocaría la contaminación de ríos, lagos y arroyos, además de la posibilidad de una infiltración hacia las aguas subterráneas y, por tanto, el deterioro del agua como recurso.

El tratamiento primario consiste en la separación física de los sólidos en suspensión, los aceites, las grasas, y, en general, la materia inorgánica que contiene la corriente de agua. En el secundario se reduce el contenido de materia orgánica acelerando los procesos biológicos naturales. Finalmente, el terciario es necesario cuando el agua va a ser reutilizada e incluye varios procesos químicos para garantizar que quede tan libre de 
impurezas como sea posible, teniendo en cuenta cuál va a ser el destino final de las aguas (Navascués y Latorre, 2013, 39).

Abordando el marco normativo, cabe mencionar que la Ley $\mathrm{N}^{\circ} 294 / 93$ "Evaluación de Impacto Ambiental" establece una obligación general para todas las actividades que generen un impacto ambiental. En sentido más específico, el Decreto № 453/13 que reglamenta la mencionada ley, incorpora en su Anexo la exigencia de la realización del EIA (Estudios de Impacto Ambiental) o un EDE (Estudio de Disposición de Efluentes) a servicios relacionados con el transporte terrestre, donde se ubican a los lavaderos. Es importante destacar que Licencia Ambiental otorgada por la autoridad administrativa ambiental MADES (Ministerio del Ambiente y Desarrollo Sostenible) constituye un requisito previo para el otorgamiento de autorizaciones del Departamento de Inspección general de los municipios, quienes cobran los cánones para habilitar un lavadero de automóvil.

Por su parte, la Resolución $\mathrm{N}^{\circ} 222 / 02$ de la Secretaría del Ambiente (SEAM, hoy MADES) "Por la cual establece el Padrón de calidad de aguas en el territorio nacional" clasifica el agua según su uso, en 4 tipos preponderantes, basado en parámetros e indicadores específicos. El artículo 7 establece los parámetros tolerados para el vertido de efluentes en los cuerpos de agua.

Para una mejor comprensión se recurre a Navascués y Latorre (2013), quienes enseñan que: Las autorizaciones de vertidos establecen las condiciones en que deben realizarse los vertidos, según la norma que le aplique, deben especificar las instalaciones de depuración necesarias y los elementos de control de su funcionamiento, así como los límites cuantitativos y cualitativos que se impongan a la composición del efluente y el importe del canon de control del vertido (p. 40).

A nivel local, la Municipalidad de Pilar no cuenta con ordenanzas que rigen sobre esta materia, solo existe una ordenanza, la $N^{\circ} 41 / 2003$ "Por lo cual se establece la obligatoriedad de la construcción de las cámaras sépticas y pozos ciegos y la prohibición del vertido de los desagües cloacales y/o aguas servidas en los pozos semisurgentes de agua”. En cuanto a la gestión del agua, la Ley No 3239 “De los Recursos Hídricos del Paraguay" establece que las aguas, superficiales y subterráneas, son propiedad de dominio público del Estado y que su acceso para la satisfacción de las necesidades básicas debe ser garantizado por el Estado, en cantidad y calidad adecuada (art. 3) y que, si bien 
su uso para fines domésticos y de producción familiar básica no está sujeto a permisos, ni concesiones, ni impuestos de ningún tipo, deberán estar inscriptos en el Registro Nacional de Uso y Aprovechamiento de los Recursos Hídricos, al solo fin de su contabilización en el Balance Hídrico Nacional (art. 15).

Además, este mismo cuerpo legal establece que será prioritario el uso y aprovechamiento de los recursos hídricos superficiales y subterráneos para consumo humano (art. 16) y establece un orden de prioridad para los demás usos, ubicando en quinto lugar a las actividades industriales (art. 18).

De ello se desprende que el uso industrial de las aguas superficiales y subterráneas está sujeto a las evaluaciones técnicas (art. 17) y que requiere necesariamente estar inscrito en el mencionado registro nacional.

La importancia de la investigación se justifica en la necesidad de contar con datos que permitan conocer el uso y aprovechamiento a los efectos de estimar la demanda del agua subterránea. Por lo expuesto, la investigación analizó el uso del agua en los lavaderos de automóviles de la ciudad de Pilar a fin de identificar la fuente de origen, determinar el tratamiento y disposición final de efluentes, así como el cumplimiento de normativas ambientales vigentes.

\section{MATERIALES Y MÉTODOS}

La investigación, de tipo descriptivo, llevada a cabo en la ciudad de Pilar, Departamento de Ñeembucú en el año 2019, analiza el uso del agua en los lavaderos ubicados en el casco urbano, para lo cual utiliza un enfoque mixto, que recolecta, analiza y vincula datos cuantitativos y cualitativos en el estudio, con diseño observacional, transversal.

A modo de caracterización del área de estudio, el clima de la zona según la clasificación climática de Thornthwaite, corresponde a un ambiente sub-húmedo - húmedo mega termal (Amabile, 2017), citado por (Chamorro, 2018)

La Ciudad de Pilar, Capital del Departamento de Ñeembucú, está ubicada en la rivera del Río Paraguay. Este departamento se caracteriza por poseer una topografía relativamente plana, haciendo que el $80 \%$ de su superficie de 1,2 millones de hectáreas de tierras esté permanentemente inundada o semi-inundada. Actualmente posee caminos de acceso en tiempos lluviosos, de manera muy limitada (Chamorro, 2018).

De la población total de 24 Lavaderos de automóviles habilitados por el Departamento de Inspección general de la Municipalidad de Pilar, se tomó una muestra de 19 lavaderos, 
determinada por el cálculo del tamaño óptimo, con un nivel de confianza del $95 \%$ y con muestreo aleatorio.

Cabe exponer lo señalado por Gamboa Graus (2017, p.10) quien explica que, en muestreos probabilísticos, además del tamaño de la población, es necesario considerar los márgenes de error que se desean tolerar. El autor recomienda incrementar el tamaño de la muestra si se requiere mayores niveles de confianza.

Se utilizaron técnicas de encuesta y observación, apoyadas en cuestionario y guía de observación. Los cuestionarios fueron aplicados a los propietarios de los lavaderos y las observaciones permitieron recabar otras evidencias.

Para cumplir con el objetivo de analizar el uso del agua en los lavaderos de automóviles de la ciudad de Pilar, se utilizaron tres variables de estudios: fuentes de suministros, tratamiento de efluentes y disposición final del mismo, y cumplimiento de normativas ambientales vigentes, específicamente la realización del EIA (Estudio de Impacto Ambiental) o un EDE (Estudio de Disposición de Efluentes) que implica contar con la Licencia Ambiental de la autoridad administrativa competente (MADES), bajo el 'régimen de la Ley $N^{\circ}$ 294/93 "Evaluación de Impacto Ambiental” y del Decreto Reglamentario $N^{\circ} 453 / 13$ en cuyo Anexo incorpora a los lavaderos de automóviles como servicios relacionados con el transporte terrestre. Por su parte, otra exigencia legal es la establecida en la la Ley No 3239 “De los Recursos Hídricos del Paraguay" para el uso y aprovechamiento del agua con fines de contabilización en el Balance Hídrico Nacional.

Por otra parte, se estimó la cantidad de agua utilizada en el proceso, basado en los datos aportados por los encuestados y en los tipos de equipamientos utilizados, así como de la cantidad de unidades lavadas. La herramienta informática de hoja de cálculo Microsoft Excel permitió organizar los datos numéricos para análisis estadísticos. Las preguntas abiertas y los resultados de las observaciones fueron examinados de manera cualitativa.

\section{RESULTADOS Y DISCUSIÓN}

La fuente mayormente utilizada es de agua subterránea y en menor proporción, del sistema de distribución centralizado de la Empresa de Servicios Sanitarios del Paraguay (ESSAP).

La extracción del agua subterránea es a través de pozos artesianos, que es una perforación vertical del subsuelo que favorece el aprovechamiento de una o más capas acuíferas 
(Gavilán, 2017). Los pozos artesianos utilizados para la extracción del agua son de mediana profundidad de entre 10 a 15 metros, equivalentes a un pozo somero.

Las aguas subterráneas como las aguas superficiales presentan riesgos y problemas en lo que se refiere a su cantidad y calidad. Según el Centro de Formación de la Cooperación Española en la Antigua Guatemala (2017), menciona estos dos aspectos:

En lo que se refiere a la cantidad, es evidente que toda extracción de agua subterránea disminuirá el flujo y el aporte de un acuífero determinado a lagos, ríos y humedales. Asimismo, los cambios en la cantidad de agua pueden afectar a su calidad, de modo que es importante calcular los tiempos de extracción para estimar los efectos diferidos de la misma y poder prever las relaciones causa efecto. Sin embargo, resulta muy difícil establecer esta relación, tanto en términos de calidad como de cantidad, puesto que los cambios se producen muy lentamente y no son inmediatamente visibles. Proteger los acuíferos no solo es importante para asegurar el suministro actual y de las generaciones futuras, sino porque todo lo que afecta a un acuífero, afecta al resto del ciclo hidrológico (p.12).

El uso desmedido o sobreexplotado de las aguas subterráneas trae consecuencias negativas para el balance hídrico del medio. Así lo manifiesta Borunda (2020) en un artículo publicado en National Geographic, hace mención que el agua subterránea cumple un papel fundamental en los ríos del mundo y mantiene el curso inclusive cuando la sequía disminuye su caudal. En el mismo artículo hace énfasis en que los seres humanos en las últimas décadas han extraído miles de millones de litros de esos embalses naturales y que entre el 15 y el 21 por ciento de las cuencas hidrográficas donde se produce extracción de agua subterránea han traspasado un umbral ecológico crítico y esa cifra podría dispararse de un 40 a 79 por ciento para el año 2050.

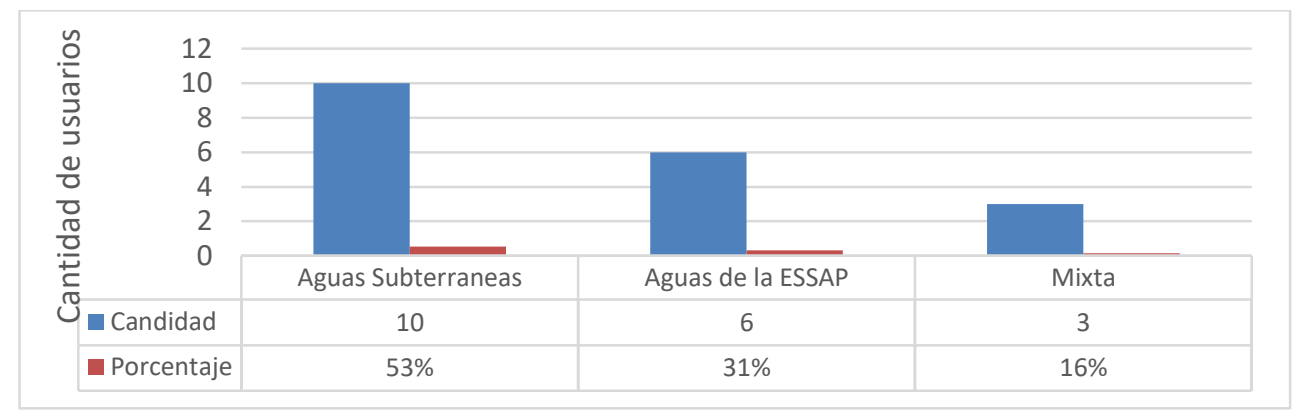

Figura 1: Origen del suministro de agua en lavaderos. Fuente: elaboración propia 
Como puede observarse en el gráfico, la práctica extractiva es frecuente en esta actividad. Es difícil saber con precisión la cantidad de agua extraída por los lavaderos que componen la muestra, no obstante, se realiza una estimación, conforme a datos extraídos de las encuestas aplicadas a los propietarios, apoyado en el marco conceptual sobre cantidad de agua según el equipo de lavado utilizado.

En su totalidad (100\%) los lavaderos utilizan mangueras con pistolas de alta presión, cuya equivalencia es de 8 lts. por minuto.

Tiempo de lavado Consumo de agua (Its.)

\begin{tabular}{|l|l|l|}
\hline Auto & 22 & 176 \\
\hline Moto & 12 & 96 \\
\hline
\end{tabular}

Tabla 1: Consumo de agua por unidad de vehículo. Fuente: elaboración propia

\begin{tabular}{|r|r|r|r|r|r|}
\hline & $\begin{array}{r}\text { Promedio } \\
\text { Semanal }\end{array}$ & Mensual & $\begin{array}{r}\text { Mensual 19 } \\
\text { lavaderos }\end{array}$ & $\begin{array}{r}\text { Consumo } \\
\text { agua (lts.) }\end{array}$ & $\begin{array}{c}\text { Total mensual } \\
\text { 19 lavaderos }\end{array}$ \\
\hline Autos & 33 & 132 & 2508 & 176 & 441.408 \\
\hline Motos & 11 & 44 & 836 & 96 & 80.256 \\
\hline & & & & & $\mathbf{5 2 1 . 6 6 4}$ \\
\hline
\end{tabular}

Tabla 2: Consumo mensual de agua en lavaderos. Fuente: elaboración propia

Otro aspecto relevante en el uso de pozos someros es la evasión del pago de tarifas de consumo. Se evidencia el consumo desmedido de aguas en el lavado de todo tipo de vehículos, ya que, al no estar sometido al control, soslaya la conciencia del usuario de practicar el uso sostenible del recurso vital, en términos económico y ambiental. Cabe mencionar que el establecimiento de tarifas constituye una herramienta de control del consumo.

Realizar el lavado con el sistema tradicional conlleva al uso deficiente del agua, considerándose un desperdicio a no aportar a cubrir necesidades básicas del ser humano o al proceso productivo. 


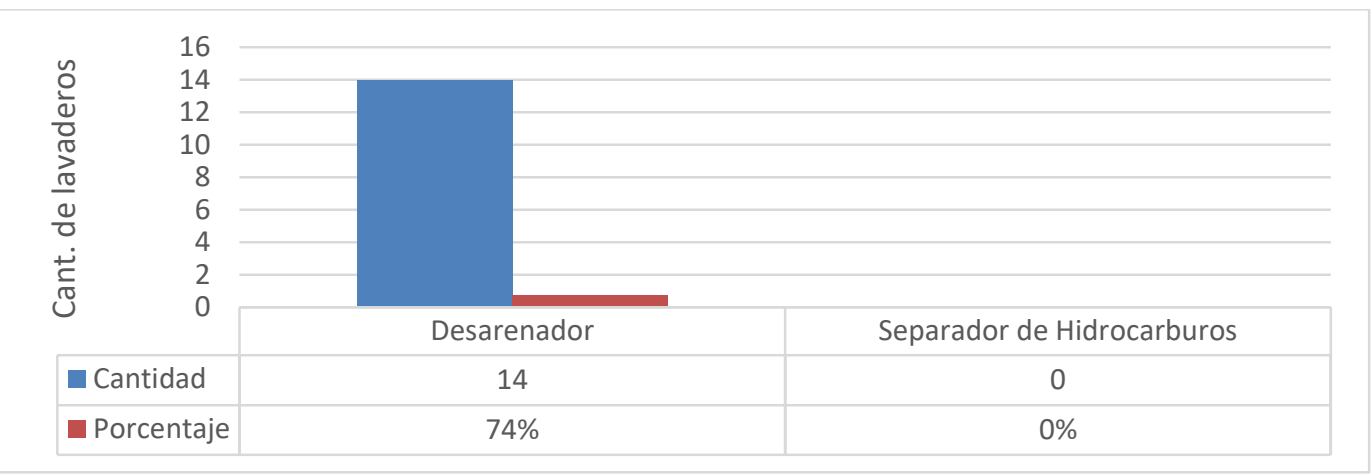

Figura 2: Tratamiento de efluentes originados del proceso de lavado de automóviles. Fuente: elaboración propia.

No se observan rejillas perimetrales anti desborde de líquidos y en la mayoría de las instalaciones observadas se evidencia el mal estado de los pisos. Este tipo de actividad requiere de piso de concreto impermeable antideslizante, considerando que puede haber infiltraciones en la napa freática.

El marco teórico hace referencia a tres tipos de tratamiento; primario, secundario y terciario. De las observaciones realizadas en las instalaciones, se pudo observar que 14 lavaderos tienen instaladas unas trampas de arena, que actúa como decantador previo de arena y lodos. Los 5 lavaderos restantes no cuentan con desarenador, para el tratamiento primario.

Navascués explica que el sistema depurador de tipo físico consta de dos fases;

En una primera columna, que contiene materiales inertes de granulometría diferenciada, quedan retenidas pequeñas partículas de sólidos en suspensión. En la segunda columna, el agua atraviesa un filtro de carbón activo donde quedan retenidos tensoactivos y otros contaminantes orgánicos. Otros son de tipo biológico donde la eliminación de materia orgánica, restos de detergente, etc., es realizada por microrganismos. Así, el agua reciclada se acumula en otro depósito desde el que se reutiliza en el lavado (2013, p.48). En cuanto al proceso de tratamiento secundario, se requiere un separador de hidrocarburos, elemento esencial en el tratamiento de aguas residuales que puedan estar contaminadas por aceites de origen mineral. El 100\% de los lavaderos, objeto de estudios, no disponen de instalaciones para el tratamiento secundario. 


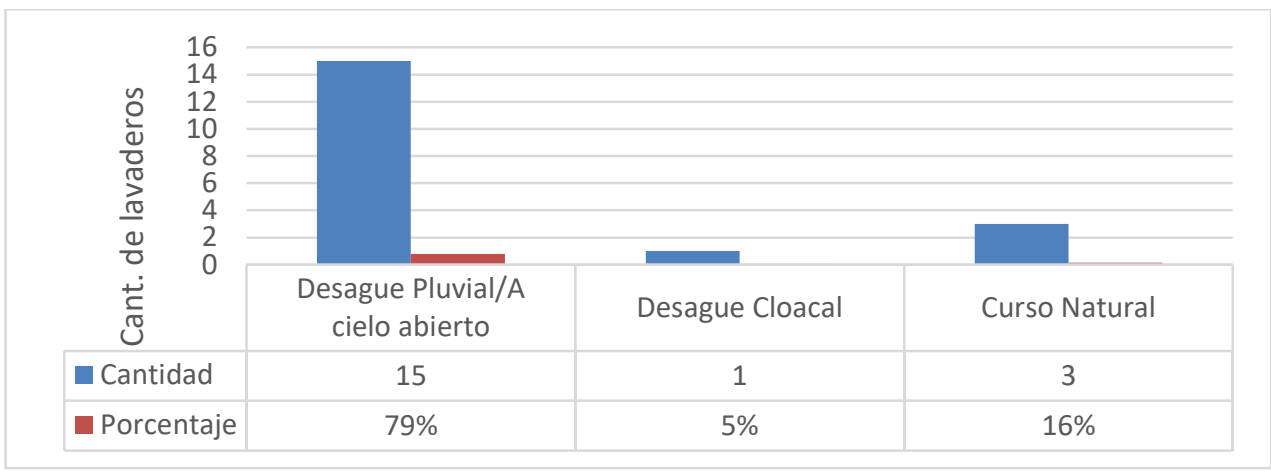

Figura 3: Disposición final de efluentes originados en lavaderos de automóviles.

Del total de lavaderos sometidos al estudio, 15 desaguan sus efluentes en canales pluviales o a cielo abierto, equivalente a un $79 \%$. Al no existir ningún tipo de tratamiento previo de las aguas residuales supone consecuencias negativas para el medio ambiente por el arrastre de distintos tipos de sustancias químicas utilizadas para el lavado como así también de los que se desprenden de los vehículos.

En este sentido, se hace necesario el desarrollo de actividades sostenibles que reduzcan el consumo de recursos y reutilicen y/o depuren los efluentes antes de devolverlos al medio ambiente. Para lograr este propósito es preciso el desarrollo de tecnologías limpias y sistemas sostenibles (Navascués y Latorre, 2013)

Los efluentes de lavaderos se componen de contaminantes (aceites y grasas, partículas sólidas, detergentes, aditivos de hidrocarburos, aceite de motor, fluido de transmisión, fluido de frenos, fluido hidráulico, anticongelante, solventes y desgrasantes)

Es importante señalar que descargar los efluentes en espacios a cielo abierto o en desagües pluviales, sin el tratamiento, supone un riesgo para la calidad de agua de las fuentes receptoras, como los cursos urbanos naturales, considerando que con las precipitaciones estos efluentes se arrastran y terminan infiltrándose en el suelo permeable, pudiendo infiltrarse hasta alcanzar el nivel de la zona de saturación que constituye las fuentes de agua subterránea, que a su vez abastece a los pozos.

En general, no se cumple con el sometimiento a Evaluación de Impacto Ambiental ni inscripción en el Registro Nacional de Uso y Aprovechamiento de Recursos Hídricos.

\section{CONCLUSIÓN O CONSIDERACIONES FINALES}

Sobre la base del marco conceptual presentado y el análisis de la información recolectada, se formulan las conclusiones y consideraciones finales.

Se pudo constatar la fuente de origen de las aguas para el lavado de vehículos es mayormente subterránea, a través de los denominados pozos artesiano, o mejor, someros. 
Al realizar el lavado manual, con mangueras con pistolas de alta presión, cuya equivalencia es de 8 lts. por minuto, se produce un consumo irracional de aguas, sumándose a esto la falta de control, tanto económico como ambiental. Cabe mencionar que el establecimiento de tarifas constituye una herramienta de control del consumo.

Por otra parte, en lo que respecta al tratamiento y disposición de sus efluentes, se evidenció que en el primer caso las formas de tratamiento son precarias, no se observan las rejillas perimetrales anti desborde de líquidos y solamente 14 de los lavadores cuentan con trampas de arena que actúan como desarenador. El resto no cuenta con desarenador para el tratamiento primario. En cuanto al tratamiento secundario y terciario no se evidenció en ninguno de los lavaderos.

En este sentido, la disposición de los efluentes es con muy poco o casi nulo tratamiento requerido para estos tipos de instalaciones, siendo que desaguan mayoritariamente en canales pluviales o en terrenos a cielo abierto, con lo cual se pone en riesgo cursos de aguas naturales.

El estudio revela que, en esta actividad, se realiza una práctica extractiva del agua, cuyo uso no es controlado, y de posibilidad de reutilización nula, ya que se devuelve en condiciones distintas a las extraídas y se pierde. Se requiere optimizar su manejo, reduciendo la cantidad de sustancias contaminantes mediante el uso de compuestos biodegradables. Los lavaderos están funcionando al margen de las normativas vigente.

El agua es un recurso que debe estar disponible no sólo en la cantidad necesaria sino también con la calidad precisa, requiere previsiones de la ordenación territorial conforme a la demanda de dinámica social. El aprovechamiento debe ser racional, previniendo derroche, contaminación y degradación para evitar alteraciones significativas en la dinámica del ecosistema. Por ello, es imperante la planificación hidrológica para el mantenimiento del Balance Hídrico y las existencias de instituciones responsables del control eficaz del uso de este recurso.

\section{LISTA DE REFERENCIAS}

Borunda, Alejandra (2020). La sobreexplotación de los acuíferos está acabando con nuestros ríos. National Geographic. Recuperado de https://www.nationalgeographic.es/ciencia/2020/03/sobreexplotacion-acuiferosesta-acabando-con-rios 
Centro de Formación de la Cooperación Española en La Antigua Guatemala (2017). Informe "La importancia de las aguas subterráneas en la gestión integrada de los recursos hídricos: aplicaciones prácticas en proyectos de cooperación internacional para el desarrollo" Recuperado de https://intercoonecta.aecid.es/Gestin\%20de1\%20conocimiento/relatoria_igme_w eb.pdf

Chamorro, L. (2018. Evaluación del efecto de la discretización espacial en el diseño de obras de drenaje urbano, mediante simulación hidrológico-hidraúlica. Caso de estudio: Cuenca del Barrio 12 de Octubre. Asunción, UNA. Recuperado de https://www.conacyt.gov.py/sites/default/files/BECA23-14_Tesis-

Liza_Chamorro.pdf

FAO (2015). Informe regional Paraguay. Organización de las Naciones Unidad para la Alimentación y la Agricultura.

Gamboa Graus, M. (2017). Estadística aplicada a la investigación educativa. Revista Dilemas Contemporáneos: Educación, Política y Valores. V (2), 5, pp. 1-32. Informe regional: América del Sur, Centroamérica y Caribe. Caso Paraguay. Año 2015.

Gavilán, Saturnino (2017). Captación de aguas subterráneas. ABC Rural. Formato digital. Recuperado de https://www.abc.com.py/edicion-impresa/suplementos/abcrural/captacion-de-aguas-subterraneas---lic-saturnino-gavilan--1576384.html

Mendieta, E. (2016). Control interno en el proceso operativo y su impacto ambiental del negocio de lavado de vehículos "Octopus" auto spa. Pontificia Universidad $\begin{array}{llll}\text { Católica del } & \text { Ecuador. }\end{array}$ https://repositorio.pucesa.edu.ec/bitstream/123456789/1699/1/76228.pdf

Navascués, E. Latorres, I. (2013). Diseño de túnel de lavado sostenible para automóviles. Universidad Pública de Navarra. Recuperado de https://core.ac.uk/download/pdf/16422465.pdf

PNUD (2006). Usos y gobernabilidad del agua en el Paraguay. Programa de las Naciones Unidas para el Desarrollo.

Tafur, J. (2017). Estrategias para disminuir el impacto ambiental de las aguas residuales industriales generadas en los lavaderos de carros. Bogotá, Fundación Universidad de América. Recuperado de 
http://repository.uamerica.edu.co/bitstream/20.500.11839/7042/1/221782-2017-

II-GA.pdf

Consejo Nacional del Ambiente, Resolución N. 04 de fecha 31 de mayo de 2005 Por la que se aprueba la Política Ambiental Nacional del Paraguay (PAN). Disponible en: http://www.seam.gov.py/gestiones-de-la-seam/politica-ambiental-nacional

Constitución Nacional del Paraguay (1992).

Convención Ramsar. Ley No 350/94 "Que aprueba la convención relativa a los humedales de importancia internacional, especialmente como hábitat de aves acuáticas". Disponible en: http://www.seam.gov.py/

Ley No 294/93 “De Evaluación de Impacto Ambiental” y el Decreto N²453/2013.

Ley $\quad N^{\circ} \quad 1.160 / 97 . \quad$ "Código Penal Paraguayo". http://www.mre.gov.py/v1/Adjuntos/Privacidad/Ley1160.pdf

Ley N 3239/2007 "De Los Recursos Hídricos del Paraguay”;

Ley N 3966/10 Ley Orgánica Municipal.

ONU. Objetivos del Desarrollo Sostenible, (ODS), [Internet] 2015 [Consultado: 30, setiembre, 2019] Disponible en: https://www.un.org

Resolución SEAM 170/06 "Por la Cual se aprueba la reglamentación del Consejo de Aguas por Cuencas Hídricas".

Resolución N²22/02 de la Secretaría del Ambiente (SEAM, hoy MADES) “Por la cual establece el Padrón de calidad de aguas en el territorio nacional 